\title{
Hybrid methodology for tuberculosis incidence time-series forecasting based on ARIMA and a NAR neural network
}

\author{
K. W. WANG, C. DENG, J. P. LI, Y. Y. ZHANG, X. Y. LI AND M. C. WU* \\ Wuxi Medical School, Jiangnan University, Wuxi, Jiangsu, People's Republic of China
}

Received 31 August 2016; Final revision 16 November 2016; Accepted 10 December 2016; first published online 24 January 2017

\section{SUMMARY}

Tuberculosis (TB) affects people globally and is being reconsidered as a serious public health problem in China. Reliable forecasting is useful for the prevention and control of TB. This study proposes a hybrid model combining autoregressive integrated moving average (ARIMA) with a nonlinear autoregressive (NAR) neural network for forecasting the incidence of TB from January 2007 to March 2016. Prediction performance was compared between the hybrid model and the ARIMA model. The best-fit hybrid model was combined with an ARIMA $(3,1,0) \times(0,1,1)_{12}$ and NAR neural network with four delays and 12 neurons in the hidden layer. The ARIMA-NAR hybrid model, which exhibited lower mean square error, mean absolute error, and mean absolute percentage error of $0 \cdot 2209,0 \cdot 1373$, and $0 \cdot 0406$, respectively, in the modelling performance, could produce more accurate forecasting of TB incidence compared to the ARIMA model. This study shows that developing and applying the ARIMA-NAR hybrid model is an effective method to fit the linear and nonlinear patterns of time-series data, and this model could be helpful in the prevention and control of TB.

Key words: ARIMA, hybrid model, NAR, tuberculosis (TB).

\section{INTRODUCTION}

Tuberculosis (TB) is a communicable infectious disease that is transmitted almost exclusively by cough aerosol, and is caused by the Mycobacterium tuberculosis complex [1]. As a major cause of illness and death, TB has plagued humankind worldwide for thousands of years and remains a serious public health problem, with more than 9.6 million new cases and 1.5 million related deaths occurring annually [2]. World Health Organization (WHO) reported that China was one of the six countries that have the largest number of TB cases, and China was among three countries

\footnotetext{
* Author for correspondence: Mr M. C. Wu, Wuxi Medical School, Jiangnan University, 1800 Lihu Road, Wuxi, Jiangsu 214122, People's Republic of China, 214122.

(Email: mcwu168@sina.com)
}

with more than half of multidrug-resistant TB cases in 2014, second only to India [2]. In China, with the growth of population, especially the increase of migrants, many areas have experienced a resurgence of TB.

The prediction of the incidence of TB as a form of early surveillance and detection can facilitate prevention and control of TB. Epidemic forecasting models were regarded as important tools to predict the occurrence of infectious diseases and to formulate reasonable precautions. Many statistical methods have been used for forecasting infectious diseases, such as exponential smoothing, the grey model, generalized regression methods, ecomposition methods, multilevel time-series models, the Markov model, and the autoregressive integrated moving average (ARIMA) model [3-7]. Of these methods, the ARIMA model is the most popular linear modelling technique for 
forecasting time series. This model assumes that the present data have a linear relationship with past data points and past errors of a time series. However, real-world problems show uncertainty and are often complex and nonlinear, therefore the linear approach might be not suitable for cases where independent and dependent variables are in a nonlinear relationship. To overcome the problem of nonlinearity, several nonlinear models have been proposed as alternative techniques, where the artificial neural network (ANN) has become one of the most popular and important methods. ANN models can approximate a nonlinear mapping with any degree of complexity and without prior knowledge of problem solving, which makes them attractive in forecasting tasks. However, the ANN model cannot capture both linear and nonlinear patterns of data equally well [8]. Recently, to overcome the limitations related to the separate use of these models in real problems, hybrid methodologies (ARIMA-ANNs) that decompose a time series into its linear and nonlinear form have recently been shown to be successful for single models [9-11]. All of these hybrid models improve the prediction accuracy of future values compared to the ARIMA model alone. Therefore, to predict the incidence of TB, a hybrid model incorporating ARIMA and a nonlinear autoregressive (NAR) network model was developed on the basis of annual and monthly TB incidence from January 2007 to March 2016 in Jiangsu province, China. The hybrid model will be helpful for forecasting the epidemic trend of TB and for providing references for TB public health interventions.

\section{METHODS}

\section{Data collection}

The observed monthly cases of TB were obtained from the website of the Bureau of Health, Jiangsu province, China, and population data was collected from the Jiangsu Statistics Bureau. In our study, we collected the incidence time series of TB from January 2007 to March 2016.

In this study, a hybrid model, developed by combining ARIMA and a NAR model, is utilized as a benchmark model. The forecasting of the hybrid ARIMA model will be accurately evaluated by comparison with the ARIMA model. To demonstrate the effectiveness of the proposed hybrid model, two datasets for use in training and forecasting are utilized in this study to examine the performance. Regarding the single ARIMA model, in the first part, 99 months' data are taken into account for the January 2007February 2015 period. These data are used in the modelling performance to construct the models. In the second part, with the help of the model constructed in the first part, the predication performance of that model is calculated using 12 months' data for the March 2015-March 2016 period.

\section{The ARIMA model}

Box \& Jenkins presented the ARIMA model in 1970 [12]. It has been widely used in financial, economic, and social scientific fields. The general form of the ARIMA models is written as follows [12]: ARIMA $(p, d, q) \times(P, D, Q)_{s}$, where $p$ is the number of parameters in the autoregressive (AR) model, $d$ the differencing degree, $q$ the number of parameters in the MA model, $P$ the number of parameters in AR seasonal model, $D$ the seasonal differencing degree, $Q$ the number of parameters in MA seasonal model, and $s$ the period of seasonality. Because there was a strong seasonality trend in this study, we constructed a seasonal ARIMA $(p, d, q) \times(P, D, Q)_{s}$ model. Prior to fitting the ARIMA model, an appropriate differencing of the series is usually performed to make the series stationary. If the series is not stationary, differencing can be used to transform it into a stationary series. The BoxJenkins approach uses an iterative model-building strategy consisting of four steps: identification, estimation, diagnostic checking, and forecasting. Box \& Jenkins proposed the use of the autocorrelation function (ACF) and the partial autocorrelation function (PACF) of the sample data as basic tools to identify the order of the ARIMA model. The conditional least squares method was applied to estimate the model parameters. The adequacy of the established model for the series is verified by employing whitenoise tests to check whether the residuals are independent and normally distributed. Several ARIMA models may be identified, and the selection of an optimum model is necessary. Such selection of models has been proposed based on Akaike's Information Criterion (AIC) and Bayesian information criterion (BIC).

\section{The NAR model}

An ANN is an intelligent nonlinear mapping system built to loosely simulate the functions of the human 
brain and has been considered as a nonlinear regression analysis tool capable of approximating any sort of arbitrary function [13]. Owing to their flexibility as function approximators, ANNs are robust methods in tasks related to time-series forecasting [14]. Among various ANNs, the NAR network, as an architectural approach of recurrent neural networks with embedded memory, represents a powerful class of models that can symbolize arbitrary nonlinear dynamical mappings and has favourable qualities for modelling dynamical systems and forecasting nonlinear time series [15]. The defining equation for the NAR model is:

$\hat{y}(t)=f(y(t-1)+y(t-2)+\cdots+y(t-d))$,

where $f$ is a nonlinear function, where the future values depend only on the regressed $d$ earlier values of the output signal.

When using a NAR network, the closed loop network is used to perform a multistep-ahead prediction. The output of the closed loop NAR network is expressed as follows:

$\hat{y}(t+p)=f(y(t-1)+y(t-2)+\cdots+y(t-d))$,

where $p$ represents the forecast steps in the future.

\section{The hybrid model}

A time series can be considered as comprising a linear autocorrelation structure and a nonlinear component. The ARIMA model and the NAR network are methodologies that predict future values using historically observed data, and are suitable for linear and nonlinear problems, respectively. According to Zhang's [16] model, we developed a hybrid model combining the ARIMA model (linear approach) and the NAR network (nonlinear approach) for our study.

It is assumed that time series are composed of a linear autocorrelation structure and a nonlinear part:

$y_{i}=L_{i}+N_{t}$,

where $y_{i}$ denotes the original monthly incidence, $L_{i}$ denotes the linear part, and $N_{t}$ denotes the nonlinear part at time $t$. The proposed methodology of the hybrid system consists of two steps. In the first step, an ARIMA model is used to predict future values at time $t$ noted, as expressed by the following equation:

$v_{t}=\mathrm{y}_{t}-\hat{L}_{t}$

where $v_{t}$ denotes the residual at time $t$ as obtained from the ARIMA model, and $\hat{L}_{t}$ denotes the forecast value by the ARIMA model at time $t$. In the second step, the NAR model is developed to model the residuals from the ARIMA model. With $n$ input nodes, the NAR model for the residuals will be:

$v_{t}=f\left(v_{t-1}, v_{t-2}, \cdots, v_{t-n}\right)+e_{t}$,

where $f$ is a nonlinear function determined by the neural network, and $e_{t}$ is the random error. Then, the combined forecast is given by the following formula:

$\hat{y}_{t}=\hat{L}_{t}+\hat{N}_{t}$

where $\hat{y}_{t}$ represents the predicted value using the hybrid model at time $t$, and $\hat{N}_{t}$ is the forecast value of equation (6).

To construct the NAR model, it is generally best to start with the neural network time-series tool, one of the graphical user interfaces (GUI) in MATLAB, which can automatically generate command-line scripts in accordance with the demand of the research. This study collected the monthly incidence of TB (98 points) for eight years (February 2008-March 2016). From this, the data were split into three blocks of (1) training the network with $70 \%$ of data (February 2008-September 2013; 68 points); (2) validation with $15 \%$ of data (October 2013-December $2014 ; 15$ points); and (3) verification with $15 \%$ of data (January 2015-March 2016; 15 points).

\section{Forecast evaluation methods}

The performance of the model is related to the similarity in the forecast values for the test data and the observed values. Three different forecast consistency measures are used for comparing the performances obtained for the ARIMA and ARIMA-NAR models: mean square error (MSE), mean absolute error (MAE), and mean absolute percentage error (MAPE). The smaller the MSE, MAE, and MAPE of the prediction model, the better its prediction accuracy. In other words, the prediction model with the smallest MSE, MAE, and MAPE can be selected as the optimum model.

$\operatorname{MSE}=\frac{1}{n} \sum_{t=1}^{n}\left(y_{t}-\hat{y}_{t}\right)^{2}$,

$\mathrm{MAE}=\frac{1}{n} \sum_{t=1}^{n}\left|y_{t}-\hat{y}_{t}\right|$, 
MAPE $=\frac{1}{n} \sum_{t=1^{\prime}}^{n} \frac{\left|y_{t}-\hat{y}_{t}\right|}{y_{t}}$.

\section{Data processing and analysis}

The ARIMA model was constructed using the appropriate module in Stata version 12.0 (StataCorp, USA). The ARIMA-NAR modelling was implemented using the Neural Network Toolbox in MATLAB v. 8.4 (R2014b). A two-sided $P$ value of $\leqslant 0 \cdot 05$ was regarded as significant.

\section{Ethical review}

The study protocol and utilization of TB incidence data were obtained from the website of the Bureau of Health, Jiangsu province, China and no ethical issues were identified. Therefore, an ethical statement was not necessary because the data are public access data.

\section{RESULTS}

\section{The best-fit ARIMA model}

Between January 2007 and March 2016, a total of 469029 TB cases were reported in Jiangsu province. Figure 1 shows the incidence rate of TB. The annual incidence decreased from $50 \cdot 87 / 100000$ in 2007 to $80 \cdot 36 / 100000$ population in 2015 , with an average annual incidence rate of $66 \cdot 21 / 100000$ population. In January, February, and March 2014, the incidence rates were $3 \cdot 58,3.64$, and $4 \cdot 70 / 100000$ population, respectively, less than three-quarters of the rates in January, February, and March 2007. Further, Figure 1 shows that the series has an obvious seasonal trend, with a higher seasonal index in March and April. The ARIMA model can be applied to analyse the time series with characteristics of seasons and tendency. The ARIMA model is fitted to a stationary time series, and seasonal data require regular and seasonal differencing to become stationary. The test timeseries data were processed by taking the first-order regular difference and the first seasonal difference to remove the growth trend and seasonality characteristics (Fig. 2). Next, the series showed no trend, and the result of the unit root test was statistically significant $(T=-18 \cdot 604, P<0 \cdot 0001)$, which confirmed that the differenced data is stationary. This suggests that it would be appropriate to consider an order $d=1$ and $s=12$ in the fitted model given by the ARIMA $(p, 1, q) \times(P, 1, Q)_{12}$. All further statistical procedures are performed on the stationary series. Figure 3 also shows that the ACF and PACF of the new data tended to be stationary after using the one-order trend difference and one-order seasonal difference. By analysing Figure 3, a series of candidate models $\left[\operatorname{ARIMA}(3,1,0) \times(0,1,1)_{12}, \quad \operatorname{ARIMA}(3,1,0) \times(0,1,0)_{12}\right.$, $\operatorname{ARIMA}(3,1,0) \times(1,1,0)_{12}, \quad \operatorname{ARIMA}(2,1,0) \times(0,1,1)_{12}$, $\operatorname{ARIMA}(2,1,0) \times(0,1,0)_{12}, \quad$ and $\operatorname{ARIMA}(2,1,0) \times$ $(1,1,0)_{12}$ ] were tested. Based on the results of the goodness-of-fit test statistics, we confirmed the optimal $\operatorname{ARIMA}(3,1,0) \times(0,1,1)_{12}$ model, which had the lowest AIC (127.3396) and BIC (140.2644) of the six candidate models (Table 1). The Ljung-Box test also shows that its residual was white noise with $Q=$ $31.4463(P=0.8311)$, indicating that the fitted data series was stationary, random, and zero-related. Figure 4 shows that the ACF and PACF of the residual sequence fell within the random confidence interval. The parameter estimate results of the ARIMA model are shown in Table 2, and all the parameter estimates were significant. Therefore, we confirmed that the best model was $\operatorname{ARIMA}(3,1,0) \times(0,1,1)_{12}$.

\section{The hybrid model}

The NAR model was selected for the prediction of TB incidence in Jiangsu province, China, because of its ability to represent nonlinear data and complex relationships. The residual series between February 2008 and March 2016 were used as the target series of the NAR model. After selecting input variables, developing the optimum NAR model requires determination of the lag, the number of neurons in the hidden layer, and the best training algorithm. Trial and error revealed that the optimum neurons in the hidden layers and the lag number were 12 and 4 based on the highest $R$ values of training, validation, and testing data subsets of $0.9489,0.5390$, and 0.3439 , respectively. The error autocorrelation function plot, which describes the relationship of the prediction errors and time, is shown in Figure 5. For a perfect prediction model, there should only be one nonzero value of the ACF, and it should occur at zero lag. This would mean that the prediction errors were completely uncorrelated with each other (white noise). In this study the correlations, except for the one at zero lag, of prediction errors fall within the $95 \%$ confidence limits around zero. Therefore, the model appears to be adequate [17]. Model performance and prediction comparison, between the forecast and measured TB incidence time series, is shown in Figure 6. The 


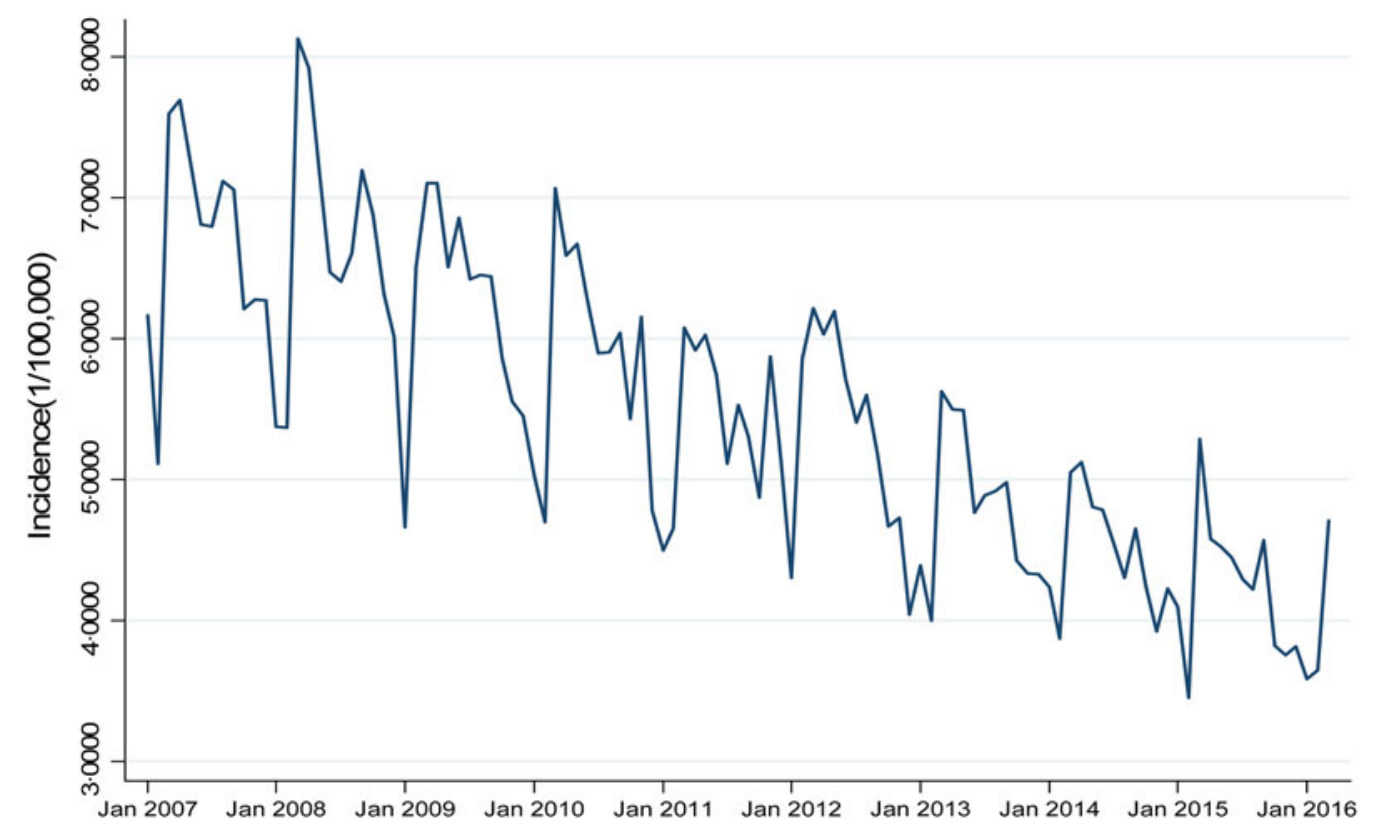

Fig. 1. The incidence/100 000 of tuberculosis from January 2007 to March 2016 in Jiangsu province, China.

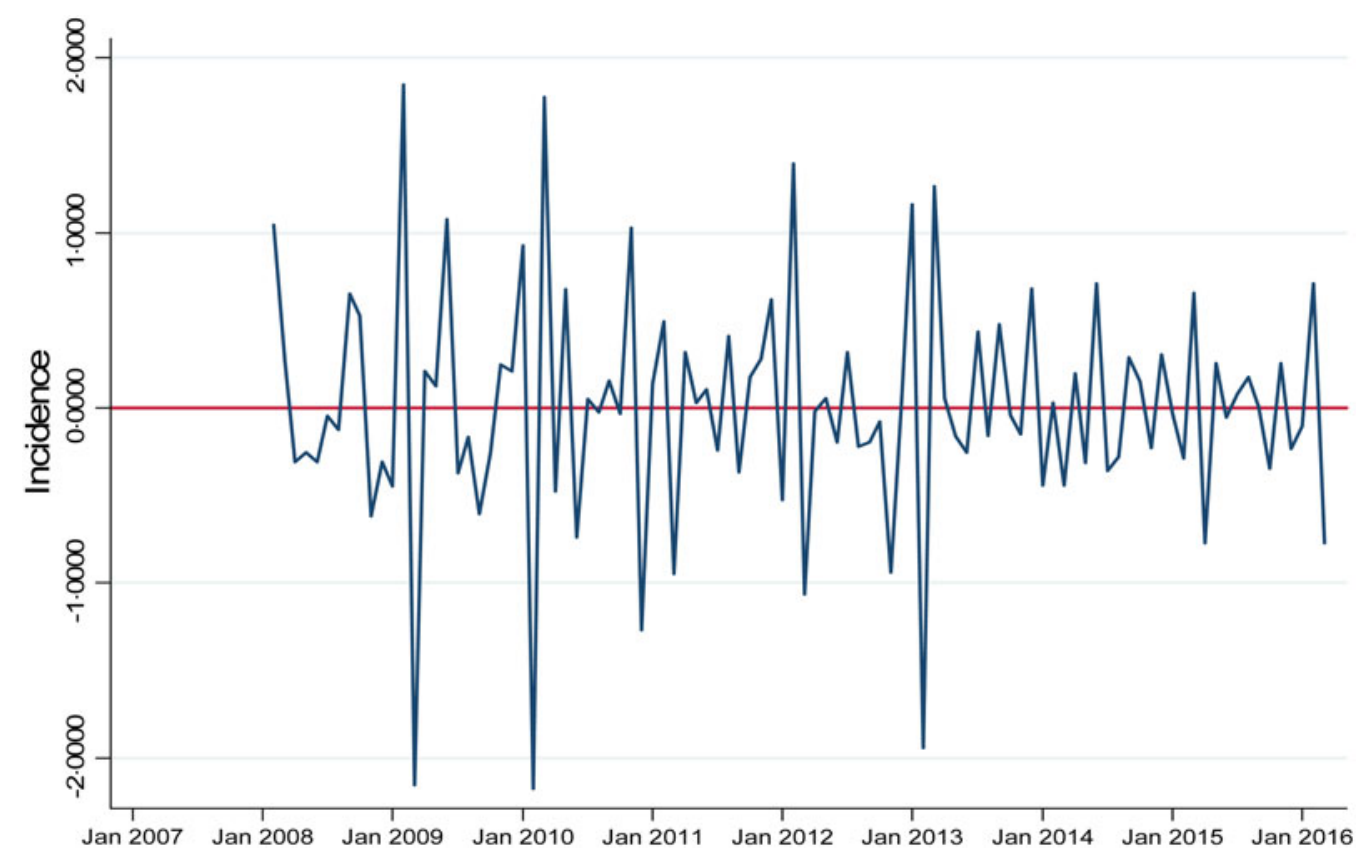

Fig. 2. One-order trend difference and one-order seasonal difference of tuberculosis incidence (per 100000 population).

response of the network, outputs, and targets was observed with the help of plots where the observed (target) values were plotted with the model calculated (output) values against time. The errors obtained in the process were also plotted against time. The overall trend of TB incidence is well captured by the model, and the model has the ability to represent and mimic the targeted output. Furthermore, most of the estimation errors vs. time lay between -1 and 1. Therefore, we determined that we had chosen the appropriate model.

\section{Comparison of the prediction accuracy by model}

Fitting and predicting curves about the incidence values for January 2007-March 2016 forecast using the 


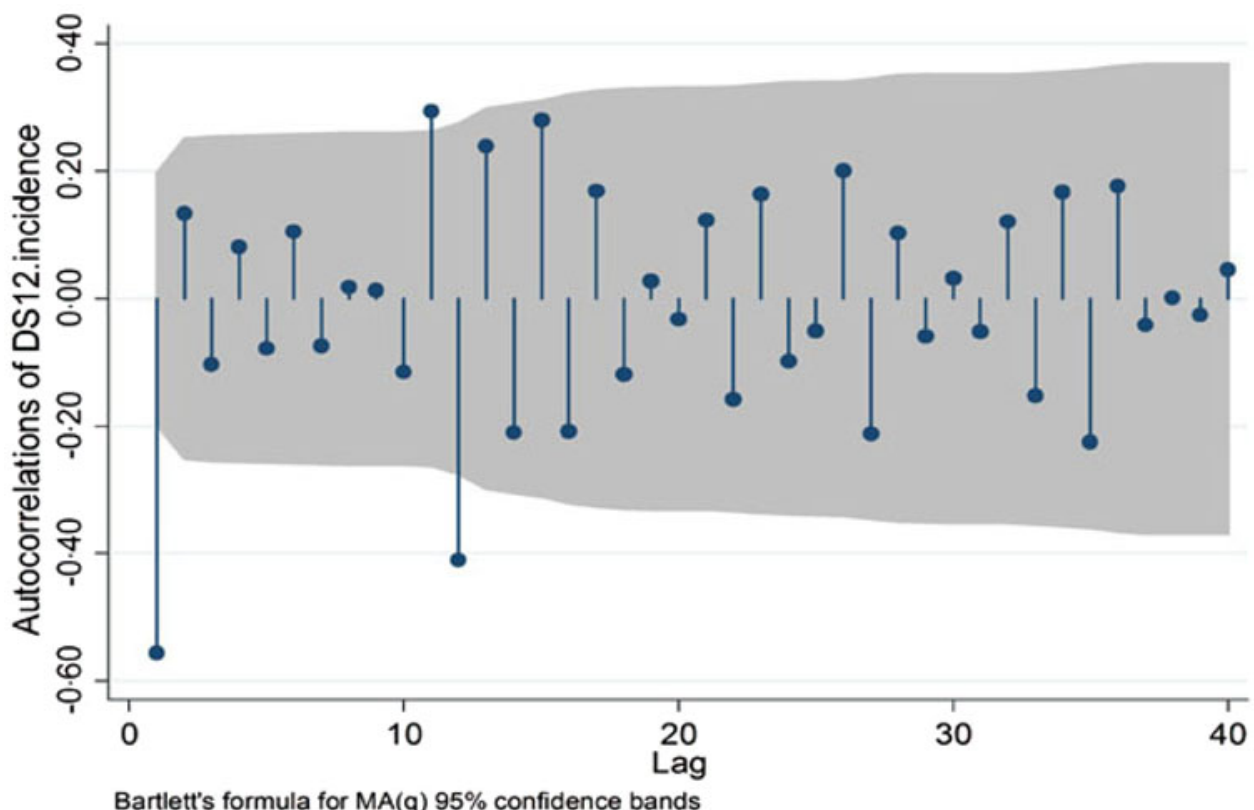

Bartlett's formula for MA(q) $95 \%$ confidence bands

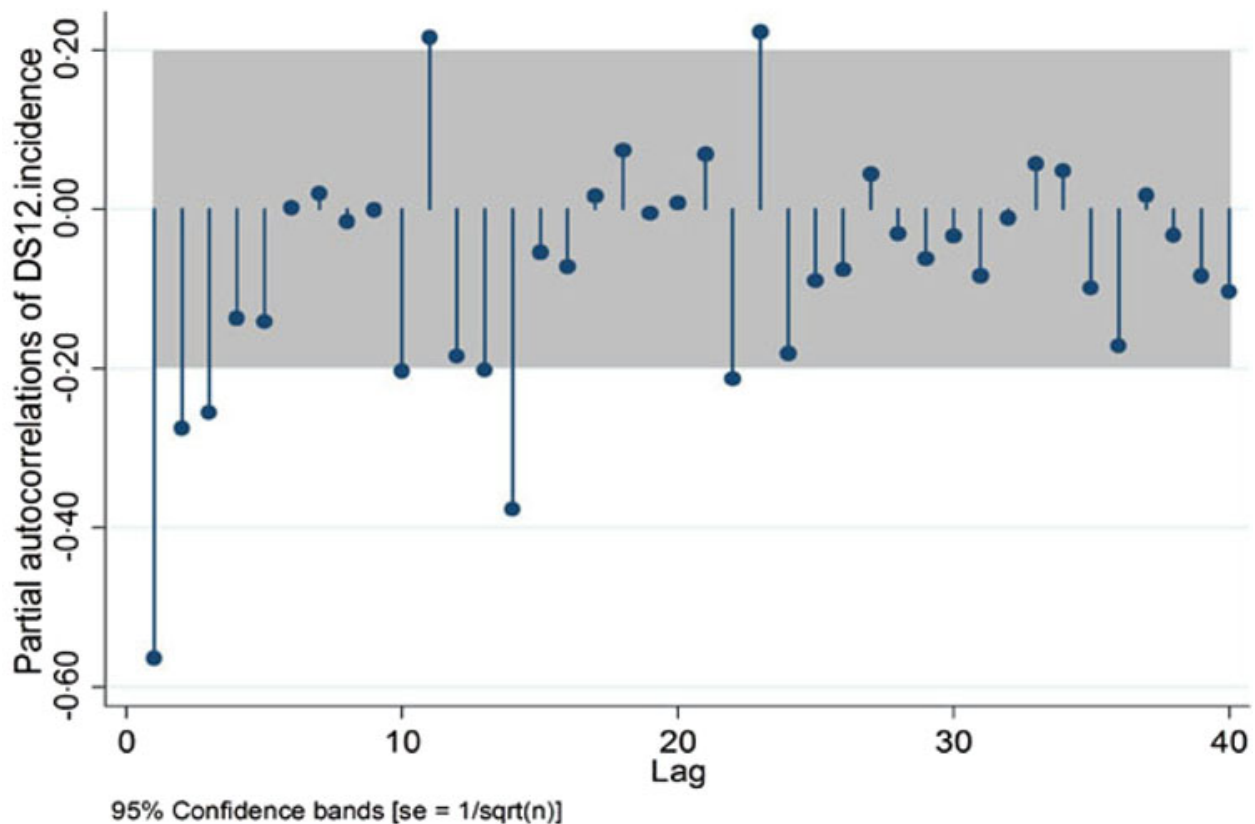

Fig. 3. Autocorrelation function and partial autocorrelation function of tuberculosis incidence after the one-order trend difference and one-order seasonal difference.

ARIMA model and ARIMA-NAR model are shown in Figure 7. It can be seen that both models are effective, but it is not obvious which model is best. To evaluate the quality of the proposed models, the MAE, MSE, and MAPE between the actual data and forecast data are chosen as the fitting and forecasting accuracy measures. As seen in Table 3, the ARIMA-NAR model's performance and forecasting for TB incidence provides more satisfactory results and better accuracy compared to the single ARIMA model. The MAE, MSE, and MAPE of the ARIMA-NAR model in the modelling stage are 0.2209 (percentage reductions, 41.84\%), 0.1373 (percentage reductions, 40.59\%), and 0.0406 (percentage reductions, $41.33 \%$ ), lower than the ARIMA model, respectively. Regarding the forecasting stage, similar results are found.

In addition, the data from January to December 2016 are used as the forecasting set (Table 4). The 
Table 1. Comparison of tested models

\begin{tabular}{|c|c|c|c|c|}
\hline \multirow[b]{2}{*}{ Model } & \multirow[b]{2}{*}{ AIC } & \multirow[b]{2}{*}{ BIC } & \multicolumn{2}{|c|}{ Ljung-Box test } \\
\hline & & & $Q$ statistic & $P$ value \\
\hline $\operatorname{ARIMA}(3,1,0) \times(0,1,1)_{12}$ & $127 \cdot 3396$ & $140 \cdot 2644$ & $31 \cdot 4463$ & 0.8311 \\
\hline $\operatorname{ARIMA}(3,1,0) \times(0,1,0)_{12}$ & $155 \cdot 3924$ & $165 \cdot 7322$ & $50 \cdot 4361$ & $0 \cdot 1248$ \\
\hline $\operatorname{ARIMA}(3,1,0) \times(1,1,0)_{12}$ & $137 \cdot 1191$ & $150 \cdot 0440$ & $38 \cdot 5409$ & $0 \cdot 5360$ \\
\hline $\operatorname{ARIMA}(2,1,0) \times(0,1,1)_{12}$ & $130 \cdot 6195$ & $140 \cdot 9593$ & $35 \cdot 5760$ & $0 \cdot 6696$ \\
\hline $\operatorname{ARIMA}(2,1,0) \times(0,1,0)_{12}$ & $160 \cdot 0286$ & $167 \cdot 7835$ & $56 \cdot 5193$ & $0 \cdot 0434$ \\
\hline $\operatorname{ARIMA}(2,1,0) \times(1,1,0)_{12}$ & $140 \cdot 5182$ & $150 \cdot 8581$ & $45 \cdot 5575$ & $0 \cdot 2519$ \\
\hline
\end{tabular}

ARIMA, Autoregressive integrated moving average; AIC, Akaike's Information Criterion; BIC, Bayesian Information Criterion.

absolute errors between actual values and predicted values fluctuate from 0.1254 to 0.3808 for the ARIMA model and 0.0021 to 0.3794 for the ARIMA-NAR model. The MAE of the ARIMANAR model is $0 \cdot 1724$, lower than the ARIMA model. Therefore the predicted values match the actual values well.

\section{DISCUSSION}

In this study, we propose to take a combination approach to time-series forecasting. A hybrid model integrating ARIMA and NAR was constructed. The ARIMA-NAR hybrid model with nonlinear components has shown better performance compared to the single ARIMA model in forecasting TB incidence in Jiangsu province, China, owing to the MSE, MAE, and MAPE measures.

The TB data series from January 2007 to March 2016 in Jiangsu province showed large fluctuating trends over a 12-month cycle with strong seasonality, as shown in Figure 1. There is a sharp rise each year that generally occurs in March and April and a drop in winter. Time-series forecasting is an important area of forecasting in which past observations of the same variable are collected and analysed to develop a model describing the underlying relationship.

Research on the model forecasting incidence can detect the epidemic tendency of TB early, which can form the basis for an early warning of the disease. The prediction model can reveal the epidemic trend of TB clearly through assumptions, parameter estimation, and fitting inspection, and can provide a theoretical basis for the development of prevention strategies and measures. Time-series forecasting is an important area of forecasting in which various factors and comprehensive effects of uncertain variables are united into a time variable. A time series needs large amounts of historical data, and identifying the relationships between them is required to develop a model for forecasting future unknown values. This method has the advantages of low-cost data collection and wide practical application. The most commonly used models for time-series analysis are ARIMA models. The popularity of the ARIMA model is due to its statistical properties as well as the well-known Box-Jenkins methodology [12] in the model building process. In this study, we obtained a multiplicative ARIMA model that can effectively extract the trend and seasonal components of time series, but the performances of modeling and forecasting were not satisfactory. As is well-known, TB is affected greatly by environmental and natural factors, for example, sunshine exposure, elevation, climate, and air pollution [18-21].

We assumed that nonlinear relationships may exist among monthly TB incidences and the ARIMA model cannot extract the full relationship efficiently. To make better predictions, an approach that uses ARIMA and ANN models together for time-series forecasts has been recommended in the present study. ANNs have a significant advantage compared to other classes of nonlinear models, in that they are universal approximators that can approximate a large class of functions with a high degree of accuracy [22]. Furthermore, ANNs belong to flexible computing frameworks for modelling a broad range of nonlinear problems and can give more efficient results in forecasting problems compared to linear models. NAR, a dynamic recurrent network with feedback connections including several layers of the network, is a powerful class of models that can symbolize arbitrary nonlinear dynamical mappings and has favourable qualities for modelling dynamic systems and forecasting nonlinear time series [23]. In this study, 


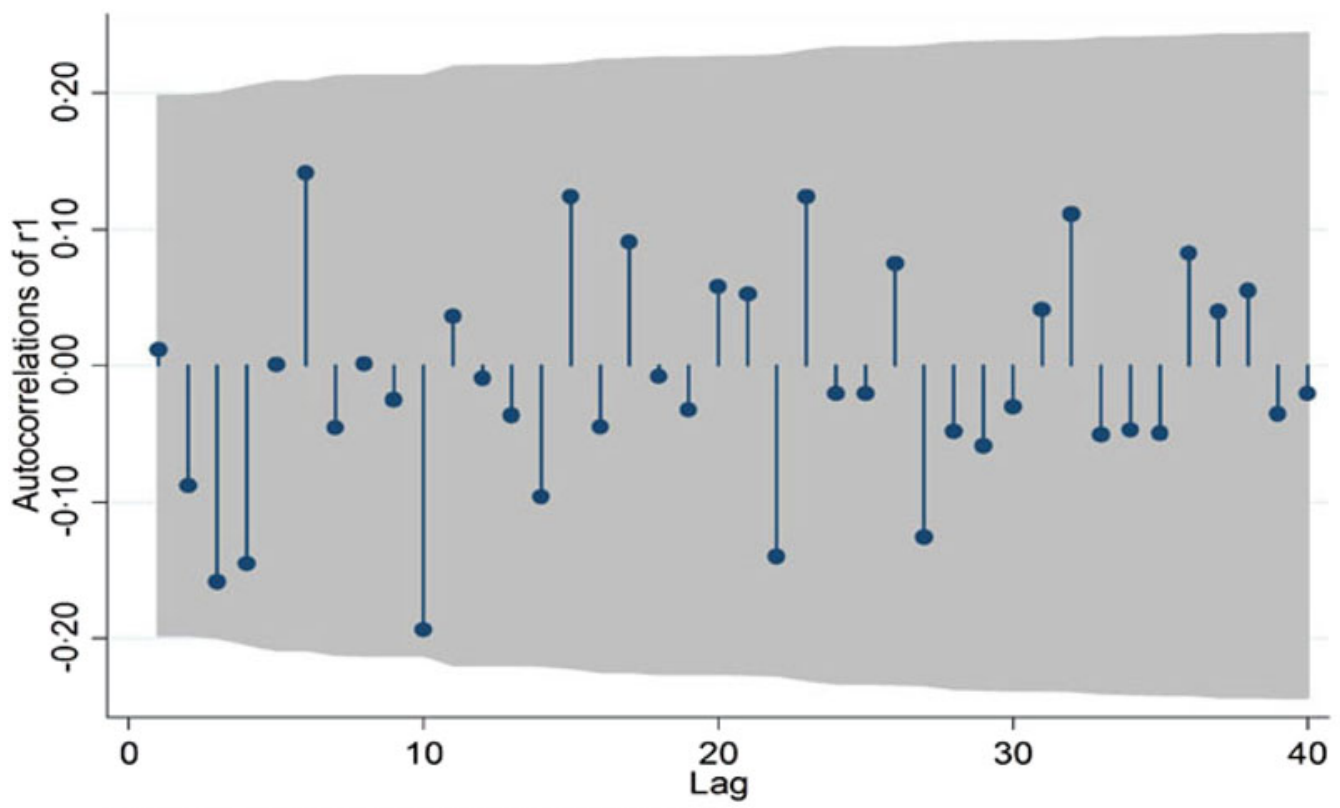

Bartlett's formula for MA(q) $95 \%$ confidence bands

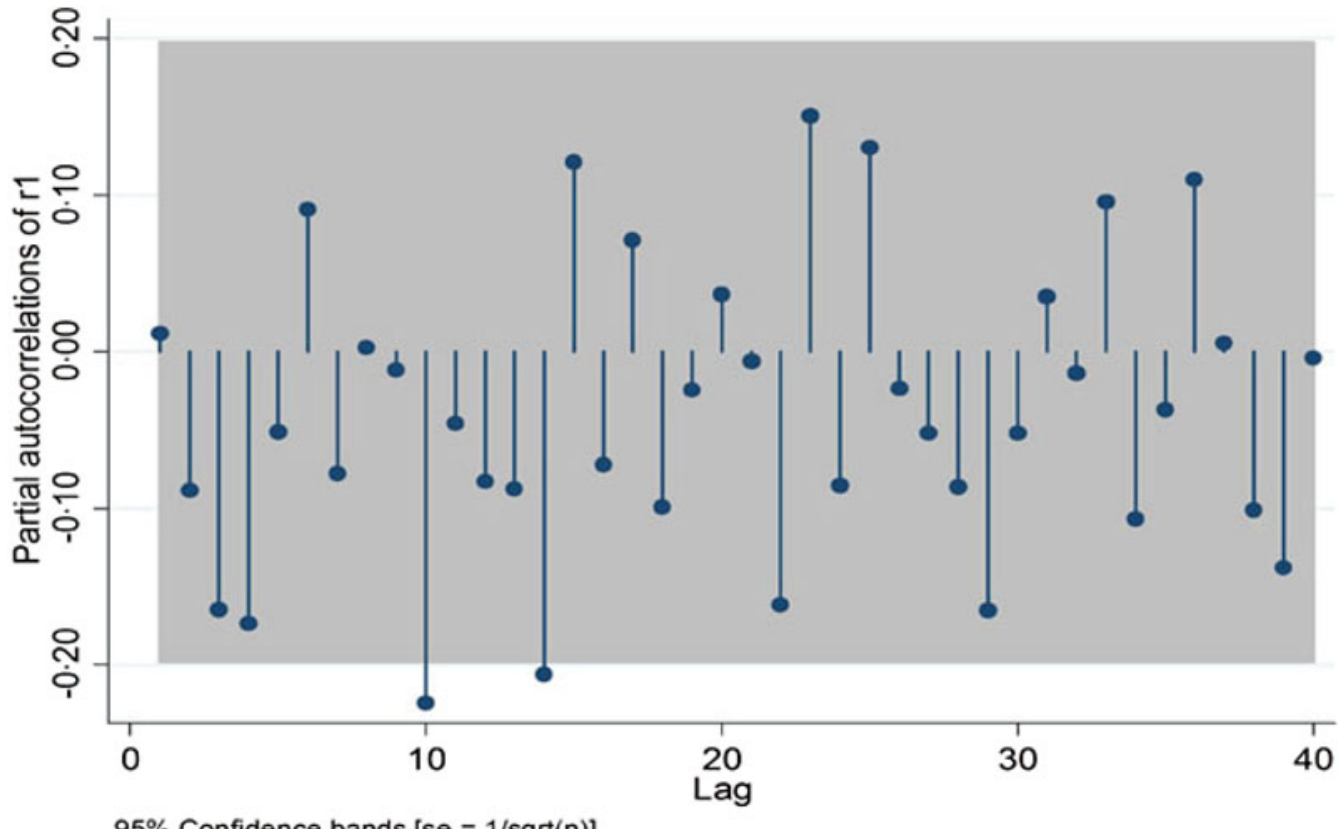

Fig. 4. Autocorrelation function and partial autocorrelation function of the residual series of the $\operatorname{ARIMA}(3,1,0) \times(0,1,1)_{12}$ model.

Table 2. Model estimation of the ARIMA $(3,1,1) \times(0,1,1){ }_{12}$ model

\begin{tabular}{|c|c|c|c|c|c|}
\hline Variable & Coefficient & S.E. & $t$ statistic & $P$ value & $95 \% \mathrm{CI}$ \\
\hline $\operatorname{AR}(1)$ & $-0 \cdot 8104$ & $0 \cdot 0772$ & $-10 \cdot 49$ & $<0 \cdot 0001$ & -0.9619 to -0.6590 \\
\hline $\operatorname{AR}(2)$ & -0.5449 & $0 \cdot 1018$ & $-5 \cdot 35$ & $<0 \cdot 0001$ & -0.7446 to -0.3452 \\
\hline $\operatorname{AR}(3)$ & $-0 \cdot 2358$ & $0 \cdot 8320$ & $-2 \cdot 84$ & $0 \cdot 0050$ & -0.3989 to -0.0728 \\
\hline Seasonal MA(1) & $-0 \cdot 2836$ & $0 \cdot 0988$ & $-2 \cdot 87$ & $<0 \cdot 0001$ & -0.8832 to -0.4267 \\
\hline
\end{tabular}

ARIMA, Autoregressive integrated moving average; CI, confidence interval; AR, autoregression; MA, moving average. 


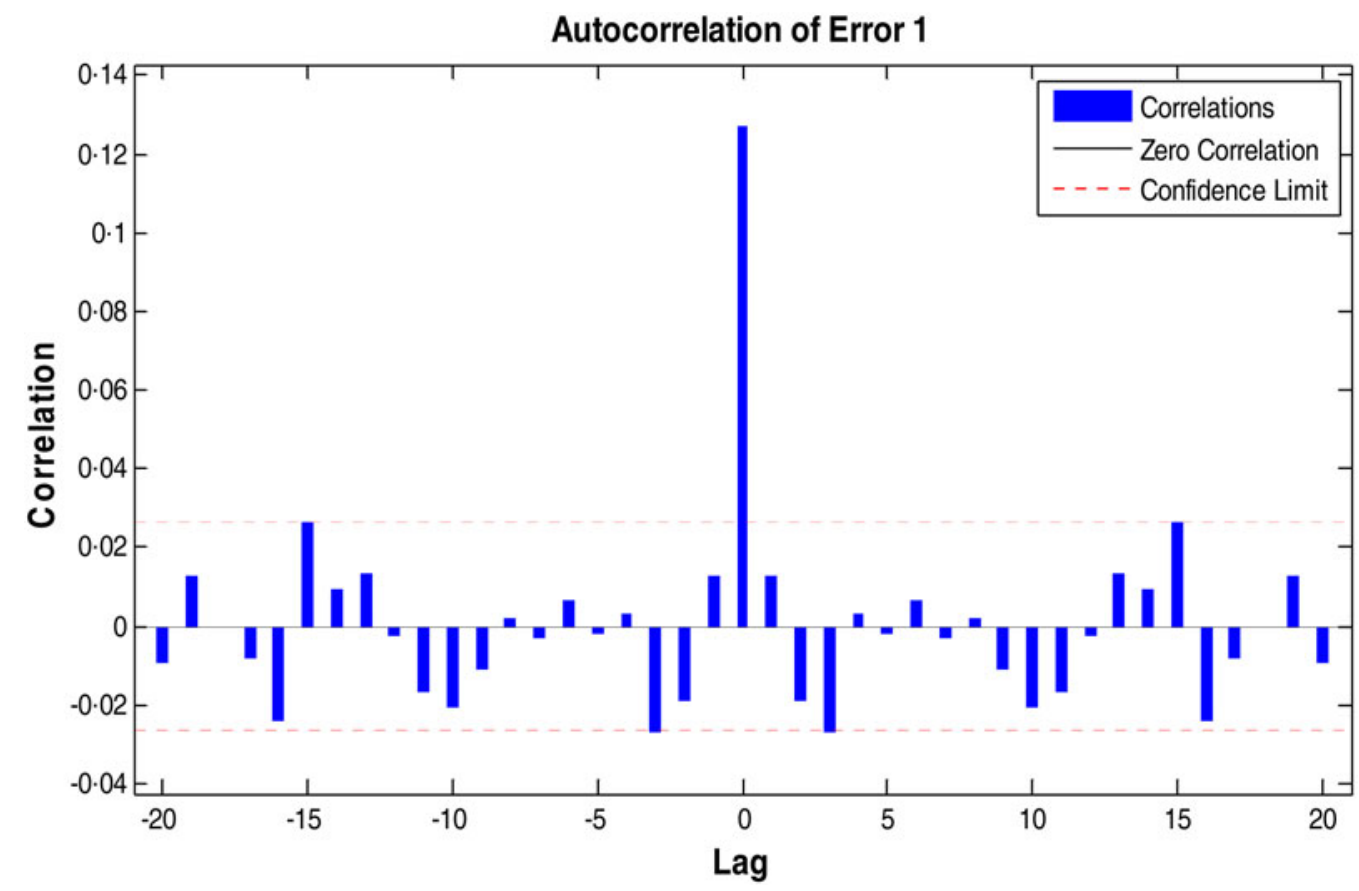

Fig. 5. The error autocorrelation plot.

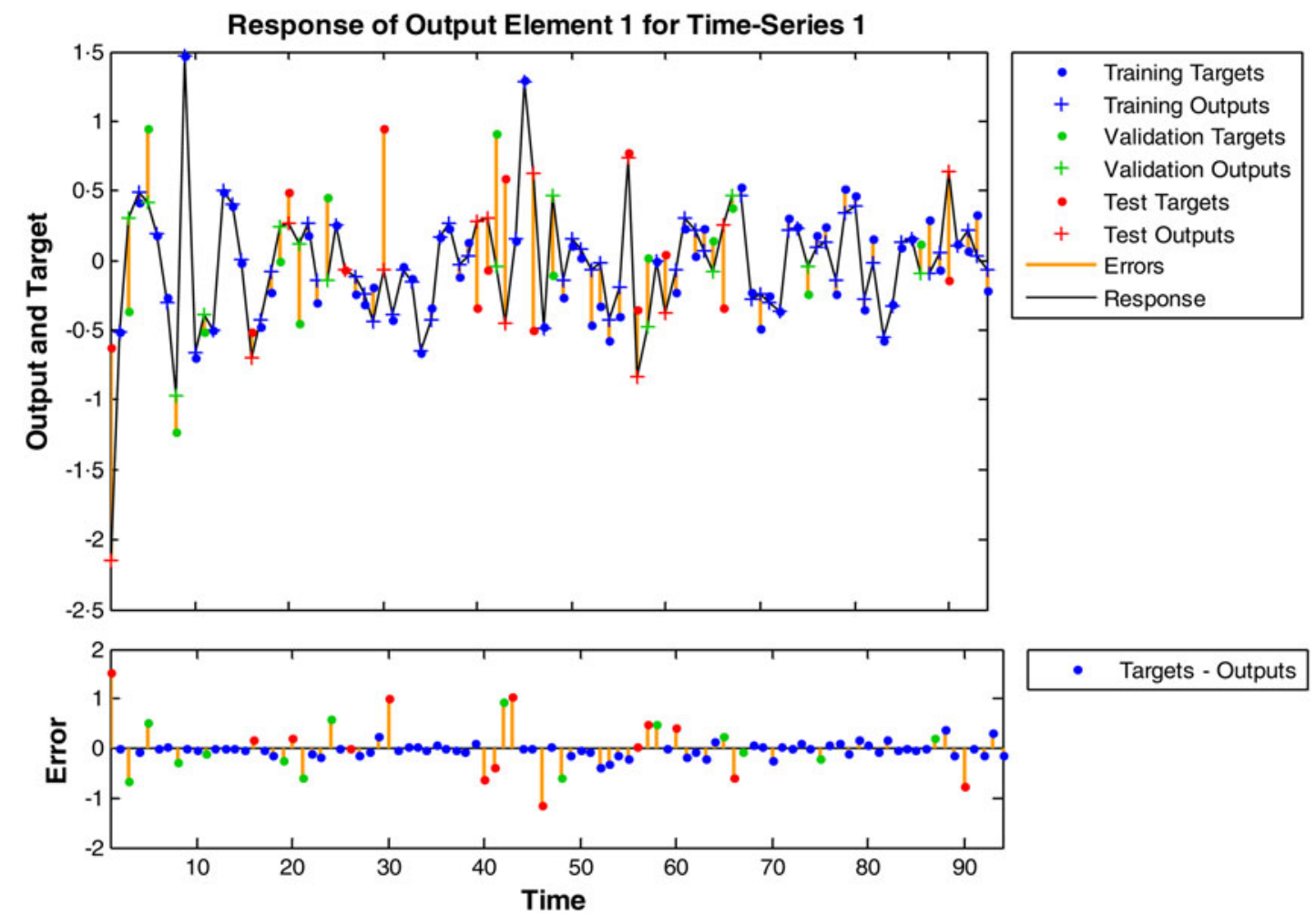

Fig. 6. Time-series response plots of different target series of ARIMA-NAR model.

the hybrid model integrated the ARIMA and NAR model, and was tested with raw seasonal data to forecast a seasonal time series. In this hybrid mechanism, the linear correlation structure of the time series is modelled by ARIMA, and then, the remaining residuals, which contain only the nonlinear part, are 


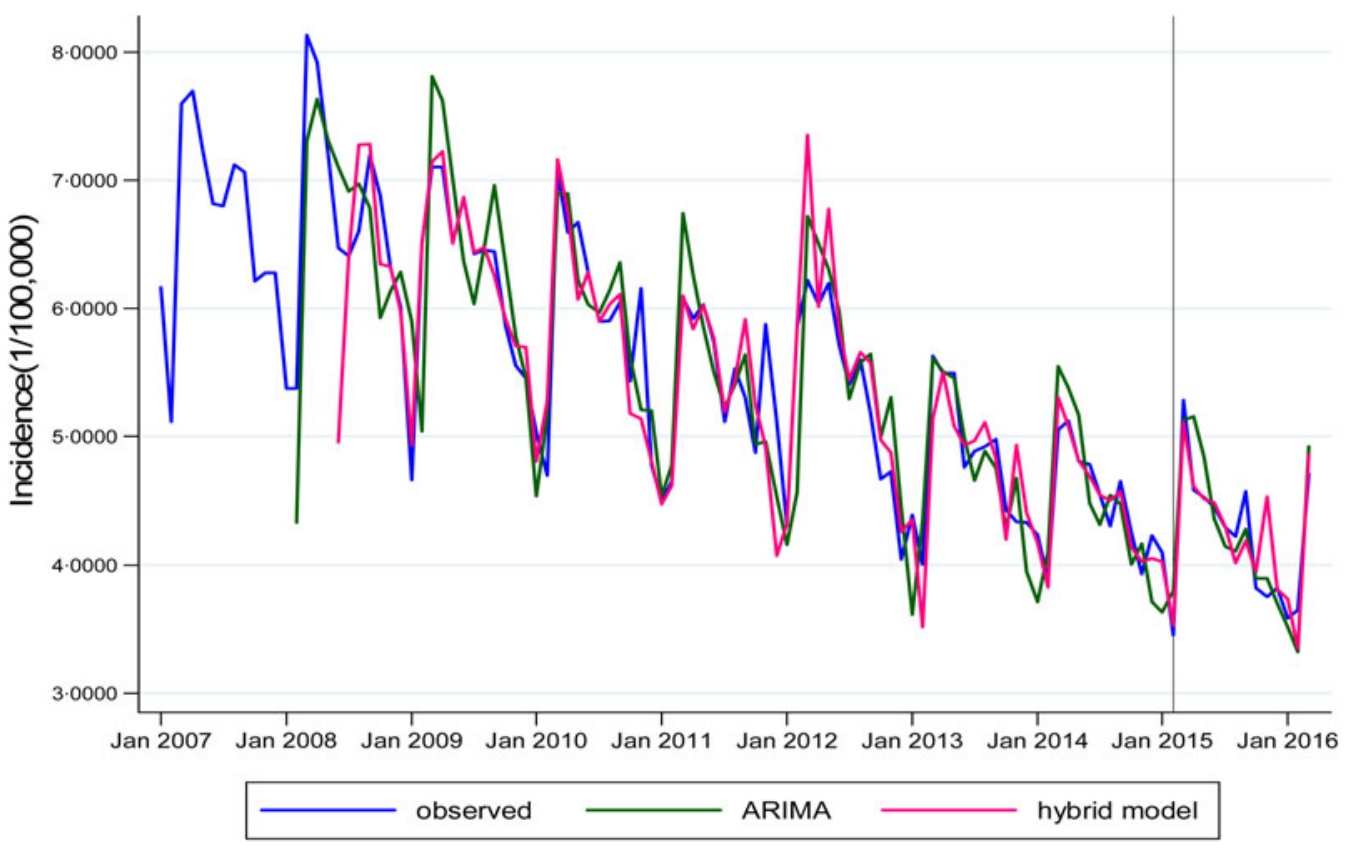

Fig. 7. Graphical presentation of different forecasting methods.

Table 3. Comparison of the modeling and forecasting performance of ARIMA and ARIMA-NAR models

\begin{tabular}{llllllll}
\hline \hline & \multicolumn{2}{l}{ Modelling performance } & & \multicolumn{2}{l}{ Forecasting performance } \\
\cline { 2 - 3 } \cline { 7 - 8 } Model & MAE & MSE & MAPE & & MAE & MSE & MAPE \\
\hline ARIMA & $0 \cdot 3798$ & $0 \cdot 2311$ & $0 \cdot 0692$ & & $0 \cdot 2129$ & $0 \cdot 0643$ & $0 \cdot 0509$ \\
ARIMA-NAR & $0 \cdot 2209$ & $0 \cdot 1373$ & $0 \cdot 0406$ & & $0 \cdot 1758$ & $0 \cdot 0517$ & $0 \cdot 0432$ \\
Reduced percentages & $41 \cdot 84 \%$ & $40 \cdot 59 \%$ & $41 \cdot 33 \%$ & & $17 \cdot 43 \%$ & $19 \cdot 60 \%$ & $15 \cdot 13 \%$ \\
\hline \hline
\end{tabular}

ARIMA, Autoregressive integrated moving average; NAR, nonlinear autoregressive; MAE, Mean absolute error; MSE, mean square error; MAPE, mean absolute percentage error.

Table 4. Comparison of predicted values and actual values for the single ARIMA model and ARIMA-NAR model during April-October 2016 (per 100000 population)

\begin{tabular}{|c|c|c|c|c|c|}
\hline \multirow[b]{2}{*}{ Month } & \multirow[b]{2}{*}{ Actual value } & \multicolumn{2}{|l|}{ ARIMA model } & \multicolumn{2}{|c|}{ ARIMA-NAR model } \\
\hline & & Predicted value & Absolute error & Predicted value & Absolute error \\
\hline April & $4 \cdot 8713$ & $4 \cdot 6646$ & $0 \cdot 2067$ & $4 \cdot 6461$ & $0 \cdot 2252$ \\
\hline May & $4 \cdot 7146$ & $4 \cdot 5892$ & $0 \cdot 1254$ & $4 \cdot 7167$ & $0 \cdot 0021$ \\
\hline June & $4 \cdot 5353$ & $4 \cdot 3700$ & $0 \cdot 1653$ & $4 \cdot 3618$ & $0 \cdot 1735$ \\
\hline July & $4 \cdot 5311$ & $4 \cdot 2098$ & $0 \cdot 3213$ & $4 \cdot 3464$ & $0 \cdot 1847$ \\
\hline August & $4 \cdot 5890$ & $4 \cdot 3470$ & $0 \cdot 2420$ & $4 \cdot 2096$ & $0 \cdot 3794$ \\
\hline September & $4 \cdot 3900$ & $4 \cdot 5840$ & $0 \cdot 1940$ & $4 \cdot 4476$ & 0.0576 \\
\hline October & $3 \cdot 6432$ & $4 \cdot 0240$ & $0 \cdot 3808$ & $3 \cdot 8275$ & $0 \cdot 1843$ \\
\hline MAE & & & $0 \cdot 2336$ & & $0 \cdot 1724$ \\
\hline
\end{tabular}

ARIMA, Autoregressive integrated moving average; NAR, nonlinear autoregressive; MAE, Mean absolute error. 
modelled by the NAR model. Moreover, this study compares the results obtained from the hybrid model with the forecast results from the single ARIMA model. It is found that the ARIMA-NAR hybrid model outperformed the single ARIMA mode in terms of overall proposed criteria, including MSE, MAE, and MAPE. In the present study, the MSE, MAE, and MAPE of the ARIMA-NAR model were lower than those of the single ARIMA model in the forecasting and modelling stages. The NAR model can learn to predict a simple time series given past values of the same time series. Some advantages of the NAR model with a gradient-descending learning algorithm have been reported: (1) learning is more efficient in NAR networks than in other neural networks and (2) the NAR model generalizes better and converges much faster than other networks [23]. Therefore, the experimental results suggested that the proposed ARIMA-NAR hybrid model, which can extract the linear and nonlinear components of TB incidence in Jiangsu province, China, is typically a reliable tool for forecasting similar problems.

\section{CONCLUSIONS}

In this paper, a new hybrid forecasting model is proposed by integrating an autoregressive integrated moving average (ARIMA) model and a nonlinear autoregressive network (NAR) for TB incidence timeseries forecasting. The ARIMA and NAR models are used together, with the aim of capturing the component of the time-series data and the nonlinear component. The MSE, MAE, and MAPE were used as performance criteria to measure the goodness-of-fit of ARIMA and hybrid models. The experimental results showed that the ARIMA-NAR hybrid model is superior to the single ARIMA model in this inspection data series. It is believed that the prediction results and the comments presented in this paper will be helpful to policy makers in China for the prevention and control of TB.

\section{ACKNOWLEDGEMENTS}

We thank the Bureau of Health, Jiangsu province, China, and the Jiangsu Statistics Bureau. This study was supported by the young teachers' startup fund for scientific research at Jiangnan University (JUSRP11569), the plan of public health research centre of Jiangnan University (JUPH201508) and the project of Wuxi science and technology supporting plan (WX0302-B010507-150016-PB).

\section{DECLARATION OF INTEREST}

None.

\section{REFERENCES}

1. Dheda K, et al. Tuberculosis. Lancet 2016; 387: 12111226.

2. World Health Organization. Global TB report 2015. Geneva: World Health Organization, 2015. (http://www. who.int/tb/publications/global_report/en/). Accessed 4 December 2015).

3. Tabaszewski M, et al. Using a set of $\operatorname{GM}(1,1)$ models to predict values of diagnostic symptoms. Mechanical Systems and Signal Processing 2015; 52-53: 416-425.

4. Zhang X, et al. Applications and comparisons of four time series models in epidemiological surveillance data. PLoS ONE 2014; 9: e88075.

5. Yang W, et al. Comparison of filtering methods for the modeling and retrospective forecasting of influenza epidemics. PLoS Computational Biology 2014; 10.

6. Li Q, et al. Application of an autoregressive integrated moving average model for predicting the incidence of hemorrhagic fever with renal syndrome. American Journal of Tropical Medicine and Hygiene 2012; 87: 364-370.

7. Li K, et al. Daily temperature change in relation to the risk of childhood bacillary dysentery among different age groups and sexes in a temperate city in China. Public Health 2015.

8. Taskaya-Temizel T, et al. A comparative study of autoregressive neural network hybrids. Neural Networks 2005; 18: 781-789.

9. Aslanargun A, et al. Comparison of ARIMA, neural networks and hybrid models in time series: tourist arrival forecasting. Journal of Statistical Computation and Simulation 2007; 77: 29-53.

10. Jain A, et al. Hybrid neural network models for hydrologic time series forecasting. Applied Soft Computing 2007; 7: 585-592.

11. Yu L, et al. Application of a new hybrid model with seasonal auto-regressive integrated moving average (ARIMA) and nonlinear auto-regressive neural network (NARNN) in forecasting incidence cases of HFMD in Shenzhen, China. PLoS ONE 2014; 9: e98241.

12. Box GEP, Jenkins G. Time Series Analysis, Forecasting and Control. San Francisco, CA: Holden-Day, 1970.

13. Adly AA, et al. Utilizing neural networks in magnetic media modeling and field computation: a review. Journal of Advanced Research 2014; 5: 615-627.

14. Connor JT, et al. Recurrent neural networks and robust time series prediction. IEEE Transactions on Neural Networks 1994; 5: 240-254.

15. Benmouiza K, Cheknane A. Small-scale solar radiation forecasting using ARMA and nonlinear autoregressive 
neural network models. Theoretical \& Applied Climatology 2016; 124: 945-958.

16. Zhang GP. Time series forecasting using a hybrid ARIMA and neural network model. Neurocomputing 2003; 50: 159-175.

17. Beale MH, et al. Demuth. Neural Network Toolbox ${ }^{\mathrm{TM}}$ Getting Started Guide. The MathWorks Inc. 2012.

18. Koh GC, et al. Tuberculosis incidence correlates with sunshine: an ecological 28-year time series study. PLoS ONE 2013; 8: e57752.

19. Tanrikulu AC, et al. Tuberculosis in Turkey: high altitude and other socio-economic risk factors. Public Health 2008; 122: 613-619.
20. Li XX, et al. Seasonal variations in notification of active tuberculosis cases in China, 2005-2012. PLoS ONE 2013; 8: e68102.

21. Tremblay GA. Historical statistics support a hypothesis linking tuberculosis and air pollution caused by coal. International Journal of Tuberculosis and Lung Disease 2007; 11: 722-732.

22. Zhang GP. A neural network ensemble method with jittered training data for time series forecasting. Information Sciences 2007; 177: 5329-5346.

23. Lin $\mathbf{T}$, et al. Learning long-term dependencies in NARX recurrent neural networks. IEEE Transactions on Neural Networks 1996; 7: 1329-1338. 